\title{
Renal calcified mass misdiagnosed as a renal calculus in an adult with tuberculosis "autonephrectomy": a case report Amie Victoria Clifford ${ }^{1}$, Aidan Paul Noon ${ }^{2}$, Daniel Raw ${ }^{3}$ and James Hall ${ }^{4}$
}

\author{
Addresses: ${ }^{1}$ The Medical School, University of Sheffield, Beech Hill Road, Sheffield, S10 2RX, UK \\ ${ }^{2}$ The Department of Urology, Royal Hallamshire Hospital, Glossop Road, Sheffield, S10 2JF, UK \\ ${ }^{3}$ The Department of Radiology, Barnsley District General, Gawber Road, Barnsley, South Yorkshire, S75 2EP, UK \\ ${ }^{4}$ The Department of Urology, Royal Hallamshire Hospital, Glossop Road, Sheffield, S10 2JF, UK \\ Email: AVC - mda05avc@shef.ac.uk; APN* - aidannoon@nhs.net; DR - draw@nhs.net; JH - james.Hall@sth.nhs.uk \\ * Corresponding author
}

Received: 2 June 2009 Accepted: 17 July 2009 Published: 6 August 2009

Cases Journal 2009, 2:7613 doi: 10.4076/1757-1626-2-7613

This article is available from: http://casesjournal.com/casesjournal/article/view/7613

(c) 2009 Clifford et al.; licensee Cases Network Ltd.

This is an Open Access article distributed under the terms of the Creative Commons Attribution License (http://creativecommons.org/licenses/by/3.0), which permits unrestricted use, distribution, and reproduction in any medium, provided the original work is properly cited.

\begin{abstract}
Introduction: Tuberculosis was once a disease much more prominent in the minds of UK urologists. The dramatic reduction in incidence following the success of antituberculous therapy has meant that new generation surgeons have little or no experience of the effects and management of tuberculosis of the kidney. With concern over multidrug resistant tuberculosis, human immunodeficiency virus associated tuberculosis and immigration of persons from areas endemic with this disorder, clinicians may see an increase in cases of renal tuberculosis. Renal tuberculosis "autonephrectomy" is the end stage of chronic renal tuberculosis infection and results from the caseous necrosis and progressive cavitation of the kidney. Resultant calcification may mimic the appearances of a renal calculus on plane film X-ray. Back, flank and abdominal pain are non-specific symptoms often investigated by General Practitioners using plane film X-ray. Clinicians not considering a diagnosis of renal tuberculosis may confuse the radiographic appearances with that of a renal calculus as occurred in our case. Once a diagnosis of tuberculosis autonephrectomy is made the next decision is whether any further investigations and treatment is necessary as the condition has been reported to be a cause of hypertension and reactivation of tuberculosis is also possible.
\end{abstract}

Case presentation: We describe the case of a 66 year old Caucasian female who presented to her General Practitioner with left sided lumber and loin pain. A lumbar spine X-ray showed a calcified mass reported as a renal calculus. Urological opinion was sort and a computerised tomogram confirmed a renal tuberculosis "auto nephrectomy". The patient had been diagnosed with tuberculosis aged 16. The patient had no lower urinary tract symptoms and normal urinalysis. Although there is some evidence to suggest nephrectomy is beneficial in treating hypertension in these patients (the patient in our case was on two anti hypertensive preparations), the patient did not want to consider surgery as her symptoms had settled spontaneously.

Conclusion: Although very rare in non endemic countries clinicians still need to consider a diagnosis of renal tuberculosis in patients with previous tuberculosis exposure and calcification of the urinary tract. In cases of uncontrolled hypertension consideration should be given to nephrectomy in cases of end stage renal tuberculosis. This decision should be made in consultation with a nephrologist. 


\section{Introduction}

The estimated tuberculosis (TB) incidence in the UK for 2007 was 15 per 100000 population with a prevalence of 12 per 100000 population [1]. Genito-urinary TB accounts for $15-20 \%$ of cases of extrapulmonary TB [2], of these about $25 \%$ will have had known pulmonary TB at some stage. Symptoms typically include dysuria, frequency and haematuria, which may be macroscopic or microscopic. Back, flank and abdominal pain may also be present. However there may be a long latency period before clinical manifestation of renal tuberculosis occurs. Following pulmonary primary infection there is a haematogenous phase where the glomerular and peritubular capillary bed of the kidneys become seeded with $M$ tuberculosis $[2,3]$. The bacilli may then proliferate in this region and the resulting cortical granulomas may remain dormant for many years. However a break down of host immunity may result in reactivation of these granulomas, which then enlarge, coalesce and as a result of capillary rupture then spread to the proximal tubule and loop of Henle. Through this process of granuloma formation, caseous necrosis and cavitation the entire kidney can be destroyed leading to "auto-nephrectomy" (as in our case) $[2,3]$.

Diagnosis of acute renal TB rests on three first-morningvoid urine samples. An acid-fast stain and mycobacterial culture are then performed. The results may also be supported by imaging findings. A plain abdominal X-ray may show enlargement of a kidney and calcification of the kidneys and lower urinary tract [3]. Computed tomography (CT) may help to detect renal TB and any extra-renal spread. It is also the most sensitive method of identifying renal calcification [4]. However ultrasound can also reveal calcifications, renal contractions and scars. Based on the imaging appearance of renal TB the differential diagnosis includes chronic pyelonephritis, papillary necrosis, medullary sponge kidney, caliceal diverticulum, renal cell carcinoma, transitional cell carcinoma and xanthogranulomatous pyelonephritis [2].

The management of acute renal TB requires referral to a TB expert and in many cases where the kidneys are functioning, adequately draining, and having only small cavities and scars - an intensive course of antituberculous drug therapy will be sufficient to sterilize the renal lesion and will not require the attention of a urologist [2].

\section{Case presentation}

A 68-year-old Caucasian lady presented with left sided upper abdominal pain and left renal angle pain to her GP. The GP ordered lumbar X-rays of the spine, which had shown a possible $7 \mathrm{~cm}$ renal stone (Figure 1). The pain had been occurring for several months and there were no

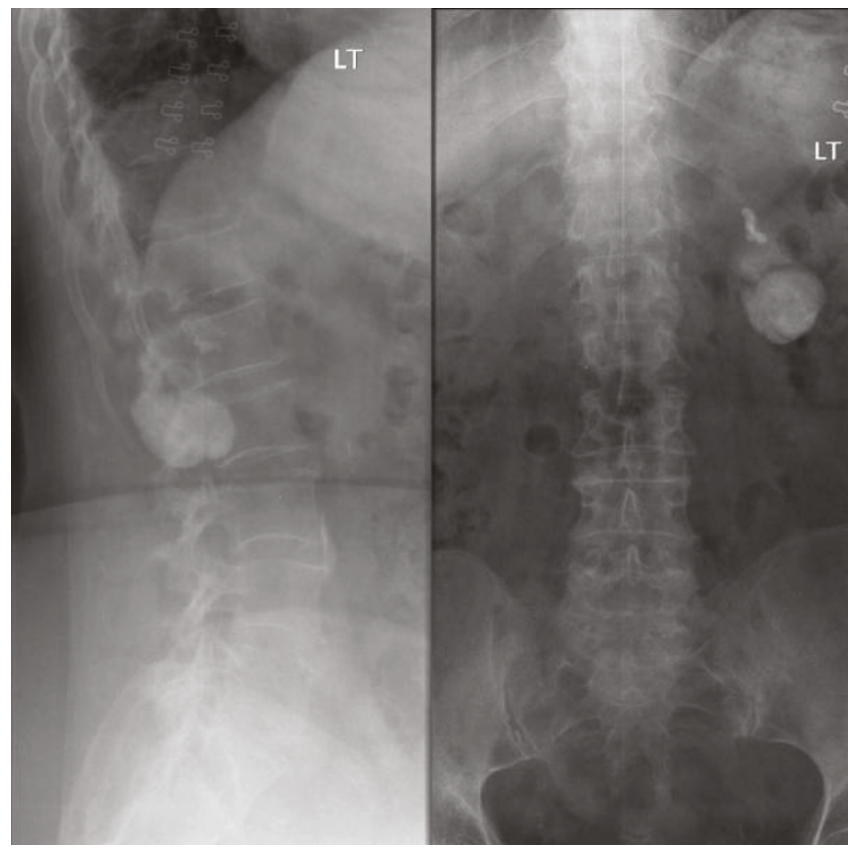

Figure I. AP \& Lateral lumbar spine X-rays.

exacerbating or relieving factors. The pain did not radiate and was not severe. She had no lower urinary tract symptoms importantly no haematuria or dysuria. Other medical problems included rheumatoid arthritis, SLE and controlled hypertension. She had no previous urological or surgical history. She was referred to a urologist for an opinion.

Laboratory investigations showed a serum creatinine of $119 \mathrm{mg} / \mathrm{dl}$ and a urea of $8.1 \mathrm{mg} / \mathrm{dl}$. Urinalysis was normal and her blood pressure was 140/80 mmHg. Her symptoms of back pain had subsided and on examination she had no loin pain. She was subsequently sent for a CT scan, which revealed that the substance of her left kidney had almost totally been replaced by a dense calcification. There was also calcification of the distal ureteric wall on the same side. Both of these findings are evident from the reconstructed CT image (Figure 2). When she was subsequently reviewed again in clinic, it came to light that she had had TB in her chest at the age of 16. A diagnosis of TB "auto nephrectomy" was made. From the time of her original referral she had no further episodes of pain and it was felt that her symptoms had been due to musculoskeletal problems and not due to the TB kidney. A review of the present literature suggested that nephrectomy may be considered for patients that are hypertensive. Our patient had well controlled hypertension and after discussion with the patient it was not felt that nephrectomy was warranted. She was discharged back to her GP, with advice to monitor her blood pressure and renal function. 


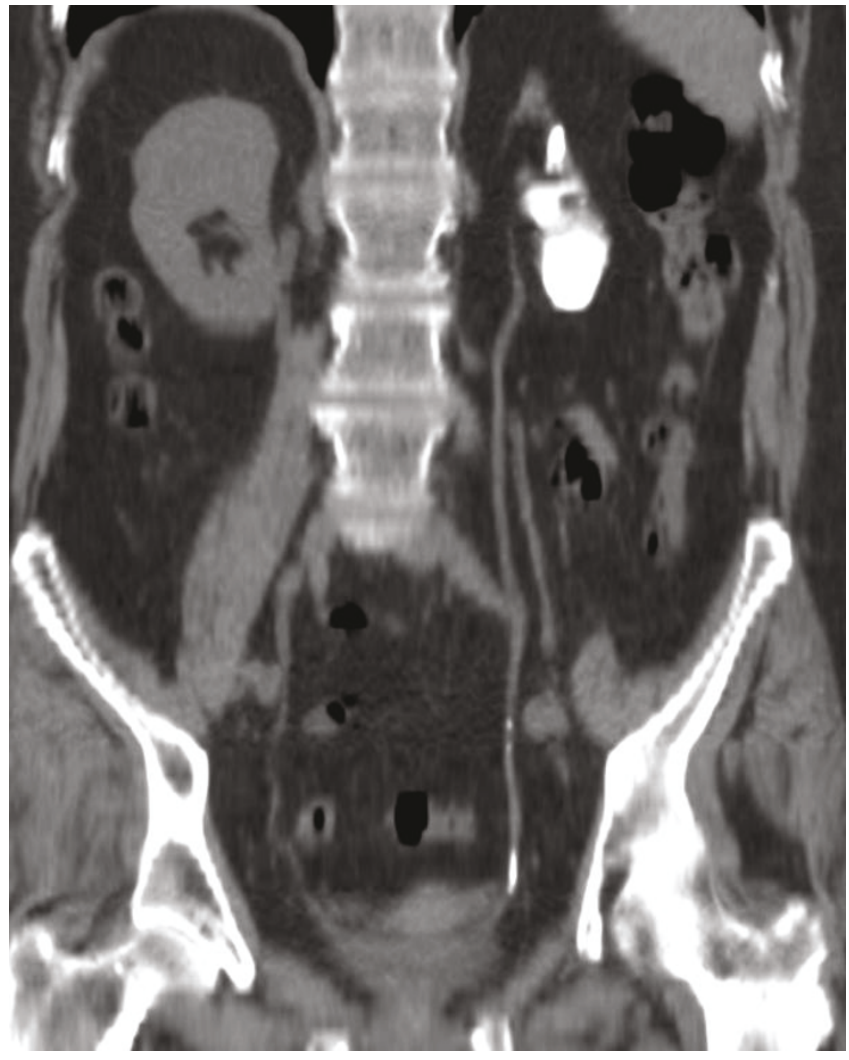

Figure 2. Non-contrast CT, coronal curves reformat.

\section{Discussion}

What to do surgically with $\mathrm{TB}$ autonephrectomy is controversial. One school of thought is that a 2-year course of medical treatment will sterilize the end stage kidney [2], possibly preventing the risk of reactivation. Butler and O'Flynn [5] in a series of 838 cases of genitourinary tuberculosis reported reactivation of genitourinary tuberculosis in only 53 of these cases $(6 \%)$ and recommended life-long follow up. However others authors have proposed that a secondary nephrectomy should be performed in order to prevent reactivation and shorten the duration of medical therapy [2].

Carl and Stark [6] recommended that if the kidney is completely destroyed by caseous decay a secondary nephrectomy is unavoidable however this is a debatable point if the kidney is non functioning and symptomless. It has been demonstrated that hypertensive patients with unilateral tuberculous nephropathy may benefit in particular from a nephrectomy [5]. In a study by Flechner and Gow [7] 17 hypertensive patients with non-functioning or poorly functioning kidneys underwent a nephrectomy which resulted in a significant reduction in blood pressure (at least a $25 \mathrm{mmHg}$ systolic and $10 \mathrm{mmHg}$ diastolic decrease) for 11 of these patients (64.7\%). Nine of these patients were followed up for at least 3 years, none of which deteriorated or were on antihypertensives.

With the development of antituberculous drugs nephrectomy has become less important. However a nephrectomy may still be valuable in the following circumstances: (i) if the kidney is calcareous, destroyed and subsequently causing pain or an abscess; (ii) the patient has hypertension alongside a unilateral renal lesion; (iii) recurrent UTI's causing persistent symptoms; (iv) suspicion of malignancy in one of the kidneys which has been damaged by renal TB. Furthermore they are the cause of (i) abscess formation with cutaneous sinus tracts and (ii) systemic hypertension which are the complications that may occur in the end stage non- functioning kidney. It is unlikely that chemotherapy alone prevents these complications from occurring as both are associated with sterile organs [5].

A series by Flechner and Gow [7] reviewed 300 cases of genitourinary tuberculosis. 73 of these patients had a standard lumbar nephrectomy following 6 weeks of antituberculous chemotherapy because an excretory urogram had shown them all to have unilateral nonfunctioning or poorly functioning kidneys. There was no perioperative mortality. All the patients received postoperative observation and follow up for a mean time of 9.4 years and urine samples did not yield mycobacteria. There were four patients who had a unilateral nonfunctioning kidney and did not have a primary nephrectomy. Three of these four patients subsequently had delayed complications however the fourth was lost to follow up.

\section{Conclusion}

Although very rare in non endemic countries clinicians still need to consider a diagnosis of renal TB in patients with previous TB exposure and calcification of the urinary tract. For patients with suspected active disease they should be seen by an expert in TB management. Where there is evidence that the disease is end stage such as "autonephrectomy" often no treatment is needed. In cases of uncontrolled hypertension consideration should be given to nephrectomy, similarly uncontrolled pain associated with the kidney may be an indication for nephrectomy. This decision should be made in consultation with a nephrologist. Patients that are immunocompromised should be referred to a TB expert for advice on whether reactivation is a risk.

\section{Abbreviations}

CT, computerised tomogram; SLE, systemic lupus erythematous; TB, tuberculosis; UTI, urinary tract infection. 


\section{Consent}

Written informed consent was obtained from the patient for publication of this case report and accompanying images. A copy of the written consent is available for review from the journal's Editor-in-Chief.

\section{Competing interests}

The authors declare that they have no competing interests.

\section{Authors' contributions}

AVC wrote the case report. APN reviewed the literature and helped to write the report. DR helped with the X-ray images and interpretation of these. $\mathrm{JH}$ was the senior clinician responsible for the case and reviewed and advised on the case.

\section{References}

I. Global tuberculosis database [http://apps.who.int/globalatlas/data Query/reportData.asp?rptType=I].

2. Narayana A: Overview of renal tuberculosis. Urology 1982, 19:23I-237.

3. Tanagho EA, Kane CJ: Specific infections of the genitourinary tract. In Smith's General Urology. Volume 1. I7th edition. Edited by Loeb M, Edmonson KG. New York: The Mcgraw-Hill Companies, Inc; 2008:22I-224.

4. Muttarak M, Pattamapaspong $\mathrm{N}$ : Clinics in diagnostic imaging. Singapore Med J 2004, 45:239-24I.

5. Butler M, O'Flynn J: Reactivation of genito-urinary tuberculosis: a retrospective review of 838 cases. Eur Urol 1975, I:14-17.

6. Carl P, Stark L: Indications for surgical management of genitourinary tuberculosis. World J Surg 1997, 21:505-510.

7. Flechner SM, Gow JG: Role of nephrectomy in the treatment of non-functioning or very poorly functioning unilateral tuberculous kidney. J Urol 1980, 123:822-825.

\section{Do you have a case to share?}

Submit your case report today

- Rapid peer review

- Fast publication

- PubMed indexing

- Inclusion in Cases Database

Any patient, any case, can teach us something

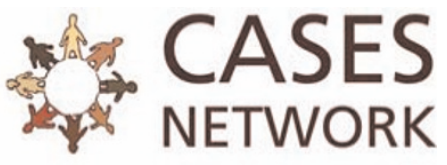

www.casesnetwork.com 